\title{
Identifying species-specific subsequences in bacteria transcription terminators- $A$ machine learning approach
}

\author{
Bao-Hua Gu${ }^{1}$, Yi Sun ${ }^{1}$ \\ ${ }^{1}$ School of Computing Science, Simon Fraser University, 8888 University Drive, Burnaby, BC, Canada. \\ Email: \{bgu, sunyi\}@,cs.sfu.ca
}

Received 24 March 2008; revised 10 March 2009; accepted 5 April 2009.

\begin{abstract}
Transcription Terminators (TTs) play an important role in bacterial RNA transcription. Some bacteria are known to have Species-Specific Subsequences (SSS) in their TTS, which are believed containing useful clues to bacterial evolution. The SSS can be identified using biological methods which, however, tend to be costly and time-consuming due to the vast number of subsequences to experiment on. In this paper, we study the problem from a computational perspective and propose a computing method to identify the SSS. Given DNA sequences of a target species, some of which are known to contain a TT while others not, our method uses machine learning techniques and is done in three steps. First, we find all frequent subsequences from the given sequences, and show that this can be efficiently done using generalized suffix trees. Second, we use these subsequences as features to characterize the original DNA sequences and train a classification model using Support Vector Machines (SVM), one of the currently most effective machine learning techniques. Using the parameters of the resulting SVM model, we define a measure called subsequence specificity to rank the frequent subsequences, and output the one with the highest rank as the SSS. Our experiments show that the SSS found by the proposed method are very close to those determined by biological experiments. This suggests that our method, though purely computational, can help efficiently locate the SSS by effectively narrowing down the search space.
\end{abstract}

\section{INTRODUCTION}

Bacterial genomes are organized into units of expression that are bounded by sites where transcription of DNA into RNA is initiated and terminated. The former site is called Transcription Promoter, which determine what region and which strand of DNA will be transcribed into RNA. The later site is called Transcription Terminator (TT), which is a stop signal to terminate the transcription process [1].

Although the mechanism of transcription termination in bacteria is rho-independent, or intrinsic, the TTs in different bacteria have a common hairpin-like RNA structure as shown in Figure 1: typically a stem-loop structure with dyadic stempairing high in guanine $(\mathrm{G})$ and cytosine $(\mathrm{C})$ residue content, followed by a uracilrich (U) stretch of sequence proximal to the termination site [2].

This particular RNA structure is encoded in the certain region of the corresponding DNA sequence. It is well known that the DNA sequences of some bacteria have species-specific subsequences (SSS) for the TTs. That is, the subsequence appears significantly more frequently in certain species than in others. For example, for bacterium Neisseria Meningitidis, the SSS is GCCGTCTGAA, while for bacterium Haemophilus Influenzae, the SSS is AAGTGCGGT [3]. Having found many DNA sequences of TTs of many bacteria, biologists are wondering whether these bacteria also have such SSS. The biological motivation behind is that the SSS might provide better understanding of the terminator evolution and how it functions in genetic exchange between pathogens.

Of course, the SSS can be determined by biological experiments. However, such experiments tend to be costly and time-consuming because every subsequence has to be examined and the number of such subsequences can be prohibitively large due to the length of the DNA sequences. To overcome the difficulty, biologists typically apply domain knowledge to narrow down the search space. However, the process of obtaining such knowledge itself would also be time-consuming and highly expensive if they are not available yet. Therefore, it is desirable to study alternative ways to effectively identify the SSS from the given DNA TT sequences. 


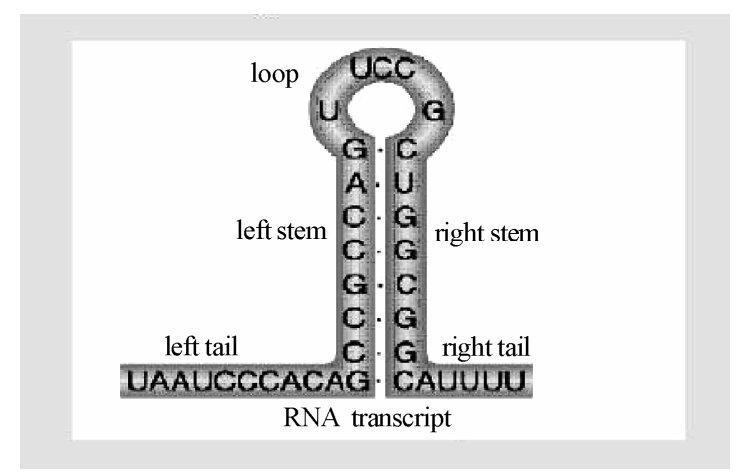

Figure 1. The stem-loop structure of bacteria transcription terminators.

In this paper we consider the problem from computational perspective. We apply machine learning techniques and propose a method to compute the SSS. Our method has three steps. In the first step, we finds out all frequent subsequences that occur more than a given threshold (or minimum support) by using generalized suffix tree. In the second step, we take the frequent subsequences as features to vectorize the DNA sequences and train an SVM classifier. In the third step, we define a measure of subsequence specificity and output the most significant features based on the measure as the SSS. Our experimental results show that the SSS found by our method are very close to the known SSS for some well known bacteria. This demonstrates that the proposed method is effective in identifying the SSS.

Our main contribution here is to introduce a machine learning approach to identifying the SSS.

We have seen successful applications of machine learning and data mining approaches to other biological problems. However, to our best knowledge, this work is the first that applies machine learning techniques to this problem. An advantage of our method is that it does not require biological domain knowledge although such knowledge would further improve the accuracy. Besides, our method does not make any assumption specific to this particular problem, and thus is potentially applicable to other similar problems.

Moreover, we also contribute to computing community by trying out new ways of using classification techniques. Traditionally, a classifier is used to predict class labels for future unseen examples. But in this paper, we use the classifier in two new ways: the tested precision of the trained classification model is used to judge whether there would be any SSS, and if so the feature weights contained in the model are used to rank the features in the model. In a word, our method can be considered as a novel application of classification to computational biology.

The rest of this paper is organized as follows: Section 2 discusses related research. The detail of the proposed method is presented in Section 3. Section 4 describes the experimental method, results and discussions. Section 5 summarizes this paper and points out our future work.

\section{RELATED WORK}

Given a bacteria DNA sequence, how to locate the segment corresponding to the RNA structure of TTs has been well studied in molecular biology and biochemistry. Interested readers are referred to [2] for research in this area. In this paper, we are dealing with a different problem: given DNA sequences that encode TTs of certain bacterium, find out whether there would be any subsequence specific to this bacterium and if so what it is. As far as we know, this problem has not been well studied in the literature of machine learning and data mining.

Support Vector Machines (SVM) has been widely studied and applied in machine leaning [4] and data mining [5] since introduced by [6]. In recent years, it has been applied to solve classification problems in biocomputing. For example, it has been used in predicting outer membrane proteins of bacteria in [7] and remote protein homology detection in [2].

We notice that in these applications, SVM lassifiers are mainly used in a traditional way, in which the classifier is built from training sequences and then applied to predicting the class labels of future unseen sequences. In our method, the classifier is used in a very different way: we use it as an indicator of whether there exist features sufficiently specific to distinguish the positive class from the negative class. If the precision of the classifier is much higher than the odds of making a random guess, we think this classification is good and reliable. Then we let the classifier to tell us which features are more important to the classification, based on which we then define the subsequence specificity measure to rank the subsequences. The most significant ones are reported as the SSS.

In our method, the DNA sequences have to be converted into vectors in the feature space solvable by SVM. The conversion is done by taking all frequent subsequences (frequent in terms of a predefined minimum number of occurrences) found in DNA sequences as features in the SVM feature space. This sort of conversion is not new, and has proved to be a very effective way to express genome/protein sequences for classification, because it is able to capture the sequential relations of the genome/protein sequences [7,8]. The frequent subsequences can be efficiently found by using generalized suffix trees.

Suffix tree is a very useful data structure for string matching problem, and has been well studied and widely applied. Interested reader are referred to [9] for more details.

\section{THE PROPOSED METHOD}

Basically, we treat the target problem of finding SSS as a classification problem. The intuition is that if there exists 
any subsequence specific to one species, then we should be able to build a good classifier to separate this species from others. Such a classifier should have contained the SSS as features. As SVM has the state-of-the-art performance for classification, we will train the classifiers using SVM.

In general, our method can be divided into three steps. In the first step, it finds out all frequent subsequences that occur more than a given threshold (called minimum support) by using generalized suffix trees. Second, it takes the frequent subsequences as features to vectorize the original DNA sequences for building the SVM classifier. If the precision of the classifier is higher than the odds of making a random guess, we think this is a good classification. Then we define a measure of subsequence specificity and output the most significant features based on the measure. We will describe each step in more detail in the following subsections.

\subsection{Frequent Subsequences Generation}

Note that SVM requires the input data to be vectorized. In this paper, the training examples are given in the form of DNA sequences, which are basically strings of letter A, C, G and T, representing the four basic amino acids of DNA. In order to convert a DNA sequence into a vector format, we need a feature space. Here we use the frequent subsequences to be the features, by which the original sequence are transformed. We hope that the subsequences can keep sufficient sequential information about the original DNA sequences.

Here is a toy example: Given 3 DNA sequences, $\mathrm{S} 1=$ ACGT, S2 = CGTA, S3 = GTAC. If the minimum support=2 (i.e., a subsequences must appear at least twice to be considered as frequent), then the frequent subsequences are $\{\mathrm{A}, \mathrm{C}, \mathrm{G}, \mathrm{T}, \mathrm{CG}, \mathrm{GT}, \mathrm{AC}\}$.

It is a nontrivial task to find all frequent subsequences from thousands of DNA sequences, each of which may contain more than hundreds of letters, because the total number of frequent subsequences could be very large. Although any methods that can output frequent subsequences from the original sequences would work as long as they are computationally feasible. To make it efficient, we make use of generalized suffix tree [9]. Suffix tree has proved to be an effective data structure for finding common substrings in linear time and linear space in terms of the total number of letters in the given string. While a generic suffix tree is built for only one string, it can be easily generalized to contain multiple strings. The generalized suffix tree provides an efficient way to finding frequent subsequences in multiple DNA sequences. Interested readers are referred to the abovementioned references for details on (generalized) suffix tree algorithms.

\subsection{DNA Sequences Vectorization}

Having obtained frequent subsequences, we take each subsequences as a feature in the feature space, and convert an original DNA sequence into a vector of the feature space.

Readers familiar with text categorization may draw an analogy from this step to the text representation, by viewing a DNA sequence as a document and a frequent subsequence as a word. Similar to text categorization, we can have different codings to represent a DNA sequence using the frequent subsequences. In our later experiments, we mainly use the binary coding: if a sequence contains a subsequences feature, it is given value "1" on that feature, otherwise, the feature value is " 0 ". This expression, though simple, proves to be very effective. It is also possible to use other expression such as TFIDF [10].

Here is a toy example (continued): following the above toy example, the three sequences can be vectorized using the binary coding. Let the feature space be the frequent subsequences set $\{\mathrm{A}, \mathrm{C}, \mathrm{G}, \mathrm{T}, \mathrm{AC}, \mathrm{CG}, \mathrm{GT}\}$, the sequence $\mathrm{S} 1=\mathrm{ACGT}$ is converted to vector $(1,1,1,1,1,1$, $1)$, $\mathrm{S} 2=$ CGTA becomes $(1,1,1,1,0,1,1)$, and the sequence $\mathrm{S} 3=\mathrm{GTAC}$ becomes $(1,1,1,1,1,0,1)$.

\subsection{SVM Classification}

SVM is based on the structural risk minimization principle from statistical learning theory introduced by V. Vapnik [6].

In its basic form, SVM learns linear decision hyperplane $h(\vec{x})=\operatorname{sign}\{\vec{w} \cdot \vec{x}+b\}$, described by a weight vector $\vec{w}$ and a threshold $\mathrm{b}$. The input is a set of $\mathrm{n}$ training example $S_{n}=\left(\left(\overrightarrow{x_{1}}, y_{1}\right), \cdots,\left(\overrightarrow{x_{n}}, y_{n}\right)\right) \in R^{N}, \quad y_{i} \in\{+1,-1\}$. For a linearly separable Sn, the SVM finds the hyperplane with maximum Euclidean distance to the closest training examples. This distance is called the margin $\delta$, as depicted in Figure 2. For a nonlinearly separable Sn, the SVM uses either a kernel function to map the original data into another dimensional space where the data points are linearly separable, or a hyperplane with a soft margin the allows some degree of training error in order to obtain a large margin. Computing the hyperplane is

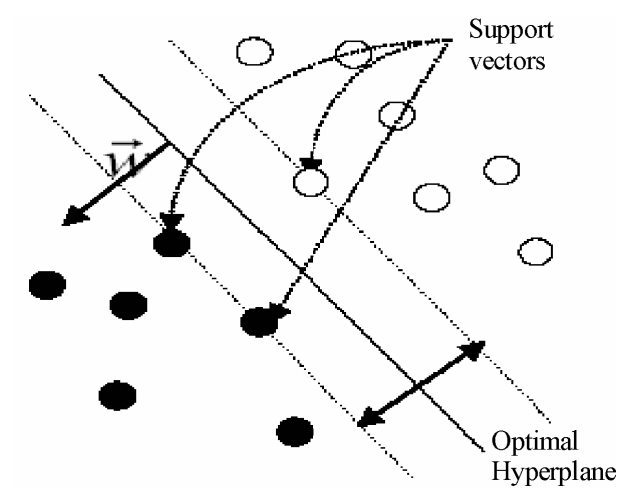

Figure 2. A linear SVM for a two dimensional training set. 
equivalent to solving an optimization problem. The resulting model is actually the decision hyperplane, expressed by $\vec{w}$ and $b$.

Note that the resulting weights can be actually viewed as a ranking of the features: a positive (negative) weight means the corresponding feature values contribute to the positive (negative) class; the larger the absolute weight value, the more important the feature is to the classification; weights of zero or very small values have little contribution to classification. Later we will use this ranking information to define what we call the measure of subsequence specificity.

We decide to use SVM for classification because it has proved to have a number of advantages over other classification algorithms. First, it can easily handle very high dimensional input space [11]. Second, it works well when the problem contain few irrelevant features [11]. Third, it does not require feature selection as a preprocessing step, because it can automatically ignore irrelevant features during the optimization [11]. Fourth, the weights contained in the resulting SVM model can be used to rank the features. All these are essential to solving the target problem.

In this paper, we use the popular SVM-light implementation of SVM [12]. Note that when training SVM classifier, one can select a kernel function suitable for the studied problem. Motivated by the fact that text documents are in general linearly separable [11], in our later experiments we only consider linear SVM model which is used by default in SVMlight.

\subsection{Measure of Good Classifiers}

Usually the performance of a classifier is measured by classification accuracy, precision and/or recall. They are defined based on a confusion matrix as shown in Table I. The Precision of the positive class is defined as $\mathrm{P}=$ $\mathrm{TP}=(\mathrm{TP}+\mathrm{FP})$, while the Recall of the positive class is defined as $\mathrm{R}=\mathrm{TP} /(\mathrm{TP}+\mathrm{FN})$. The overall Accuracy is defines as $\mathrm{A}=(\mathrm{TP}+\mathrm{TN}) /(\mathrm{TP}+\mathrm{FP}+\mathrm{TN}+\mathrm{FN})$. In our method, the performance of SVM classifier is not measured by the overall accuracy, because negative training sequences would be much more than those positives, and the overall accuracy would be heavily affected by the overwhelming negatives.

Besides, as our goal is to identify the specific subsequences in positive species, we hope to maximize the precision (i.e., the probability of the classifier making a correct prediction is high). Therefore, we will only use precision as the measure of classifier's performance. At the same time, we will report corresponding recalls for reference. Note that the precision of the classifier should be higher than the odds that one makes a random guess for the positive class label, in order to be considered as a good classification. Otherwise, the classifier does not make sense. For example, if a training data set contains
Table 1. Confusion matrix in classification.

\begin{tabular}{l|l|l}
\hline & $\begin{array}{l}\text { Actual number of } \\
\text { positive examples }\end{array}$ & $\begin{array}{l}\text { Actual number of } \\
\text { negative examples }\end{array}$ \\
\hline $\begin{array}{l}\text { Number of examples } \\
\text { classified as positive }\end{array}$ & True Positive (TP) & False Positive (FP) \\
$\begin{array}{l}\text { Number of examples } \\
\text { classified as negative }\end{array}$ & False Negative (FN) & True Negative (TN) \\
\hline
\end{tabular}

100 positive examples and 100 negative examples, if one randomly guesses for the class label of any of the 200 examples, the probability of making a correct guess is obviously $50 \%$. If a classifier than a random guess, then the classifier is useless. A bad classification may suggest that the information contains in the training examples is probably insufficient for the classifier to distinguish the positive examples from the negative examples.

In our method, we take this as an indication that the positive species probably contains no SSS. In other words, we consider a classifier is good only when its precision is much higher than the random guess odds. In our later experiments, the precision is obtained by testing against a reserved portion of the total data, which is unseen during the classifier is trained.

\subsection{Subsequence Specificity Measure}

Note that the resulting classifier will not be used as usual to predict class label for an unseen sequence. Remember the task here is to identify the SSS. For this purpose, we make use of the weights of features in the SVM model, to define what we call subsequence specificity to measure how specific a subsequence $\mathrm{f}$ is to the positive species as follows:

$$
\begin{aligned}
\operatorname{spec}(f)= & \text { svm_weight }(f) \times \operatorname{support}(f,+) \\
& \times \text { confidence }(f,+) .
\end{aligned}
$$

here weight (f) is the weight of feature f obtainable from the learned SVM model, support $(f,+)$ is a measure of how many sequences of positive species contain $f$, and confidence $(f,+)$ is a measure of how many sequences that contain $\mathrm{f}$ belong to positive species.

The definition of the subsequence specificity is based on the following observations and expectations:

1) An SSS should occur frequently in the positive species. This means it should have a high support.

2) An SSS should occur more frequently in the positive species than in the negative species. This means it should have a high confidence.

3) Having a high support and/or a high confidence does not necessarily mean a high distinguishing power, while this can be reliably judged by the feature weights in SVM model.

4) SVM may give more weight on features having low support and/or confidence. This means the weights alone are insufficient to measure the specificity.

5) Each of the three quantities alone characterizes the subsequence specificity from an unique angle. Combing 
them together should be able to give a more accurate characterization of the specificity, and thus is expected to be a more reliable measure.

\section{EVALUATION}

\subsection{The Data Set}

The data set used in this project is provided by the Brinkman Libratory of SFU Molecular Biology and Biochemistry Department. It contains known rho-independent transcription terminator sequences for 42 bacteria genomes. The source of the data set is The Institute for Genomic Research (TIGR). Each record of the data set contains one terminator sequence (including left tail, stem and loop, and right tail) and the taxonomy id of the corresponding bacterium. The total number sequences is 12763 , and 18 species have less than 100 terminator sequences.

Besides, we are also provided with a file containing the taxonomy information of all bacteria species. Figure 3 shows the taxonomy tree for the 42 bacteria, obtained from the file. The tree shows the evolution paths for the 42 bacteria. Each node in the tree represents a bacterium species, and marked by its taxonomy id. The leaf nodes are enclosed by a parenthesis. Note that among the 42 bacteria, 41 are at leaf nodes, except species 562, which has species 83334 as its children. This is the only pair of species that has a direct parent-children relationship in the given data set.

The task is to identify those DNA subsequences that are specific to certain species. The hope is that this should provide a better understanding of bacteria evolution and how it plays a role in genetic exchange between pathogens. Note that for a given species, it does not necessarily have any subsequences specific to it. Also, when talking about the specificity, one should consider the corresponding scope. That is, with respect to which species a subsequence is considered specific. For example, a subsequence can be specific to bacterium $\mathrm{A}$ with respect to another bacterium $\mathrm{B}$. Also, the subsequence can be specific to bacterium $\mathrm{A}$ with respect to all other bacteria.

Among the 42 species, we evaluate our methods on four bacteria whose SSS for the TTs are already known biologically. The four species are: Haemophilus influenzae, Neisseria meningitidis, Pasteurella multocida, and Pseudomonas aeruginosa [3]. As the SSS for the four bacteria are all found in the left inverted repeat (i.e., the left stem) of corresponding TTs, we will also use the left stem of each TT sequence to find the SSS. For each of the four species, we employ the one-againstall-others approach to build classifier by taking all its TT sequences (the left stems) as positive sequences while those of the other 41 species as negative sequences.

\subsection{Experimental Methodology}

All experiments are done using 5-fold cross validation. Averaged values over the five round experiments are reported. The linear classifiers are trained using SVMlight default settings. The frequent subsequences are obtained from the positive training sequences by setting the minimum support to be $1 \%$ of the total number of positive sequences (and the absolute support number no less than 2). These frequent subsequences represent statistically significant features with regard to the positive species, while resulting in substantially lower dimensions compared to the feature space of all potential subsequences. The classification performance is evaluated by the precisions, while the corresponding recalls are reported for reference.

\subsection{Experimental Results}

The results are given in Table II, from which we can see that the precisions for all the four species are significantly higher than the respective random guess odds. This implies that there are subsequence features that can distinguish the positive species from all the others. We then output the top 10 subsequences based on the subsequence specificity measure. The results are given in Table 2. In Table 3, the resulting subsequences that are the closest to the known SSS are underlined.

We can see that for species Pasteurella multocida and Pseudomonas aeruginosa, the underlined subsequences are exactly the same as the known SSS. For the Haemophilus influenzae and Neisseria memingitidis, our results are very close to the known SSS: the underlined ones are very close to the known SSS. In all cases, our top 10 subsequences are almost the substrings of the known SSS. This shows that our method is rather effective in finding SSSs. Note that the known SSS are found by applying biological domain knowledge [3], for example, discarding those biologically meaningless subsequences and/or limiting the length of the subsequences, which is not considered in our method. Therefore, it can be expected that our method can be improved by incorporating domain knowledge.

Table 2. Classification performance for the four bacteria.

\begin{tabular}{lcccc}
\hline $\begin{array}{l}\text { Name of the } \\
\text { Positive } \\
\text { Species }\end{array}$ & $\begin{array}{l}\text { \# of Freq } \\
\text { Subseq }\end{array}$ & $\begin{array}{l}\text { Classifier } \\
\text { Precision }\end{array}$ & $\begin{array}{l}\text { Classifier } \\
\text { Recall }\end{array}$ & $\begin{array}{l}\text { Odds of } \\
\text { Random } \\
\text { Guess }\end{array}$ \\
\hline $\begin{array}{l}\text { Haemophilus } \\
\text { influenzae }\end{array}$ & 325 & $55.81 \%$ & $14.18 \%$ & $3.19 \%$ \\
$\begin{array}{l}\text { Neisseria } \\
\text { meningitidis }\end{array}$ & 360 & $99.61 \%$ & $50.67 \%$ & $3.62 \%$ \\
$\begin{array}{l}\text { Pasteurella } \\
\text { multocida }\end{array}$ & 389 & $48.0 \%$ & $1.09 \%$ & $3.73 \%$ \\
$\begin{array}{l}\text { Pseudomonas } \\
\text { aeruginosa }\end{array}$ & 296 & $67.77 \%$ & $9.34 \%$ & $4.28 \%$ \\
\hline
\end{tabular}


Table 3. The top 10 subsequences with the greatest specificity values for the four species.

\begin{tabular}{|c|c|c|c|c|}
\hline \multirow{2}{*}{$\begin{array}{l}\text { Top spec } \\
\text { rank }\end{array}$} & \multicolumn{4}{|c|}{ The Subsequences found for each Bacterium } \\
\hline & Haemophilus Influenzae & Neisseria meningitidis & Pasteurella multocida & Pseudomonas aeruginosa \\
\hline 1 & TGCGGT & GCCGTC & TGCGGTC & GCCCCGC \\
\hline 2 & GTGCGGT & CGTCTG & GTGCGGTC & $\overline{\mathrm{CCCCGC}}$ \\
\hline 3 & GTGCGG & CGTCTGA & ACCGCAC & GCCCCG \\
\hline 4 & CGCACTT & CGTCTGAA & CCGCAC & CCCGC \\
\hline 5 & CCGCACT & GCCGTCT & TGCGGT & GCCCC \\
\hline 6 & CCGCACTT & CCGTCTG & $\underline{G T G C G G T}$ & CCCGGC \\
\hline 7 & GCGGT & CCGTCTGA & $\overline{\text { GCGGTC }}$ & GCCC \\
\hline 8 & CGCACT & CGTCT & GGCGAA & GCC \\
\hline 9 & TGCGG & CCGTCTGAA & ACCGCA & GGCGACC \\
\hline 10 & TGCGGTT & GTCTGA & CGGTC & GGC \\
\hline actual SSS & AAGTGCGGT & GCCGTCTGAA & GTGCGGT & GCCCCGC \\
\hline
\end{tabular}

\section{CONCLUSION}

In this paper, our goal is to find the species-specific subsequences for bacteria transcription terminators. By treating the problem as a classification problem, we propose a solution based on frequent subsequences and Support Vector Machines. We first find frequent subsequences from the terminator DNA sequences of the positive species. We then take all such subsequences as feature space to transform the original DNA sequences into SVM readable vectors and train SVM classifiers. The resulting classifiers are used indicators of the existence of the SSSs. In order to extract the target subsequences from the SVM model, we make use of the SVM weights on the features and define a measure called subsequence specificity. The most significant features based on the measure are output as the SSS. Our experiments show that this method is effective. As a conclusion, we have presented a novel application of classification to computational biology.

Although the proposed method is designed and evaluated on DNA terminator sequences of bacteria, we believe that it is applicable to other similar biology tasks with perhaps minor modifications. As for future work, it is desirable to evaluate the proposed method on more bacteria. Besides, the proposed method itself can be improved in many ways. For example, to refine the specificity measure to make it more accurate, and to find better methods to express DNA sequences for classification.

\section{REFERENCES}

[1] P. Turner, (2000) Molecular Biology.

[2] M. D. Ermolaeva, H. G. Khalak, O. White, H. O. Smith, and S. L. Salzberg, (2000) Prediction of transcription terminators in bacterial genomes, Journal of Molecular Biology, 301, 27-33.
[3] T. Davidsen, E. A. Rodland, K. Lagesen, E. Seeberg, and T. Rognes, (2004) Biased distribution of dna uptake sequences towards genome maintenance genes, Nucleic Acids Research, 32(3), 1050-1058.

[4] C. J. C. Burges, (1998) A tutorial on support vector machines for pattern recognition, Knowledge Discovery and Data Mining, 2(2).

[5] T. Joachims, (2002) Optimizing search engines using clickthrough data, in Proceedings of the ACM Conference on Knowledge Discovery and Data Mining (KDD-2002).

[6] V. Vapnik, (1995) The Nature of Statistical Learning Theory. Springer.

[7] S. Rong, F. Chen, K. Wang, M. Ester, J. L. Gardy, and F. S. L. Brinkman, (2003) Frequent-subsequence-based prediction of outer membrane proteins, in Proceedings of 2003 ACM SIGKDD Conference.

[8] M. Deshpande and G. Karypis, (2002) Evaluation of techniques for classifying biological sequences, in Proceedings of Pacific Asia Conference on Knowledge Discovery and Data Mining (PAKDD-2002).

[9] D. Gusfield, (1997) Algorithms on strings, trees, and sequences: computer science and computational biology, Cambridge University Press.

[10] G. Salton and C. Buckley, Term weighting approaches in automatic text retrieval, Information Processing and Management, 24(5), 1988.

[11] T. Joachims, (1998) Text categorization with support vector machines: Learning with many relevant features, in Proceedings of the European Conference on Machine Learning (ECML-1998).

[12] (2002) Svmlight support vector machine, web.

[13] B. Gu, (2007) Discovering species-specific transcription terminators for bacteria, School of Computing Science, Simon Fraser University, Tech. Rep.

[14] J. R. Quinlan, (1993) C4.5: Programs for machine learning, Morgan Kaufmann Publisher.

[15] L. Breiman, J. H. Friedman, R. A. Olshen, and C. J. Stone, (1984) Classification and regression trees, Wadsworth.

[16] H. O. Lancaster, (1969) The chi-squared distribution, John \& Sons. 